\title{
A Note On Two Operators
}

\author{
MICHAEL ELLIOTT
}

It was recently shown in [1] that, given two bounded linear operators $A$ and $B$ on a complex Hilbert space $H$, the inequality

$$
\sup _{h \in B_{H}}\|A h\|\|B h\| \geqslant \frac{1}{2}\|A\|\|B\|
$$

holds, where $B_{H}$ denotes the unit ball of $H$. Moreover, the proof of this result showed that an even stronger result is true: under the assumption that $\|A\|=\|B\|=1$, we have

$$
\sup _{h \in B_{H}} \min \{\|A h\|,\|B h\|\} \geqslant \frac{1}{\sqrt{2}} .
$$

In this note we prove that a similar result holds for any pair of operators between Banach spaces.

Theorem. Let $S$ and $T$ be bounded linear operators from one complex Banach space $X$ to another one, $Y$. Then the inequality

$$
\sup _{x \in B_{X}} \min \{\|S x\|,\|T x\|\} \geqslant \frac{1}{2} \min \{\|S\|,\|T\|\}
$$

holds (where $B_{X}$ denotes the unit ball of $X$ ).

Proof. We will assume that $\|S\|=\|T\|=1$; the general result follows easily from this special case by normalising the two operators (provided that neither of them is zero, in which case the inequality is trivial). This assumption guarantees that $X$ and $Y$ are both nonzero spaces.

Select any real number $\varepsilon$ such that $0<\varepsilon<1$. Now choose a unit vector $y \in X$ such that $\|S y\|>1-\varepsilon$. By the Hahn-Banach Theorem we can find a functional $f \in Y^{*}$ of norm 1 with the property that $f(S y)=\|S y\|$. We will show that there exist a unit vector $z \in X$ and a functional $g \in Y^{*}$ with norm 1 such that $\|T z\|>1-\varepsilon$ and $g(T z)=\|T z\|$, and which also satisfy the requirement that each of the complex numbers $f(S z)$ and $g(T y)$ has a non-negative real part. 
To find these, we begin by selecting unit elements $\tilde{z} \in X$ and $\tilde{g} \in Y^{*}$ such that $\|T \tilde{z}\|>1-\varepsilon$ and $\tilde{g}(T \tilde{z})=\|T \tilde{z}\|$. We can find a complex number $\alpha$ such that $|\alpha|=1$ and $\alpha f(S \tilde{z})|\tilde{g}(T y)|=$ $\alpha^{-1} \tilde{g}(T y)|f(S \tilde{z})|$. Indeed, if either $f(S \tilde{z})=0$ or $\tilde{g}(T y)=0$ then $\alpha=1$ will satisfy this equation; otherwise we may choose $\alpha$ to be either of the two values of

$$
\sqrt{\frac{\tilde{g}(T y)}{|\tilde{g}(T y)|} \cdot \frac{|f(S \tilde{z})|}{f(S \tilde{z})}} .
$$

Furthermore, by replacing $\alpha$ with $-\alpha$ if necessary, we can arrange that $\operatorname{Re} \alpha f(S \tilde{z}) \geqslant 0$. This guarantees that $\operatorname{Re} \alpha^{-1} \tilde{g}(T y) \geqslant 0$ also. Now let $z=\alpha \tilde{z}$ and $g=\alpha^{-1} \tilde{g}$. It is clear that $z$ and $g$ satisfy all of the conditions required of them.

Using the fact that the real part of $f(S z)$ is non-negative, we obtain

$$
\begin{aligned}
\|S(y+z)\| & \geqslant|f(S y+S z)| \\
& \geqslant \operatorname{Re} f(S y)+\operatorname{Re} f(S z) \\
& \geqslant\|S y\| \\
& \geqslant 1-\varepsilon .
\end{aligned}
$$

Similarly, from the fact that the real part of $g(T y)$ is non-negative, we may deduce that $\|T(y+z)\| \geqslant 1-\varepsilon$. With $y+z$ clearly not equal to 0 , we may define the vector

$$
x=\frac{y+z}{\|y+z\|} .
$$

The triangle inequality ensures that $\|y+z\| \leqslant 2$, and thus

$$
\min \{\|S x\|,\|T x\|\} \geqslant \frac{1}{2}(1-\varepsilon)
$$

which completes the proof.

Remark. The constant $\frac{1}{2}$ in this theorem is, in general, the best possible. This can easily be verified by considering the space $X=$ $\mathbb{C}^{2}$ equipped with the $\ell_{1}$-norm - that is, $\left\|\left(x_{1}, x_{2}\right)\right\|=\left|x_{1}\right|+\left|x_{2}\right|$ - and the two coordinate projections, $P_{1}:\left(x_{1}, x_{2}\right) \mapsto\left(x_{1}, 0\right)$ and $P_{2}:\left(x_{1}, x_{2}\right) \mapsto\left(0, x_{2}\right)$, on $X$. It is clear that $\left\|P_{1}\right\|=\left\|P_{2}\right\|=1$. Now if $\left(x_{1}, x_{2}\right)$ is a vector in the unit ball of this space satisfying 
$\left\|P_{1}\left(x_{1}, x_{2}\right)\right\| \geqslant \frac{1}{2}$, then we have $\left|x_{1}\right| \geqslant \frac{1}{2}$ and hence $\left|x_{2}\right| \leqslant \frac{1}{2} ;$ it follows that $\left\|P_{2}\left(x_{1}, x_{2}\right)\right\| \leqslant \frac{1}{2}$. This establishes the inequality

$$
\sup _{x \in B_{X}} \min \left\{\left\|P_{1} x\right\|,\left\|P_{2} x\right\|\right\} \leqslant \frac{1}{2},
$$

proving that $\frac{1}{2}$ is the best possible constant, as claimed.

\section{REFERENCES}

[1] R. Strašek and B. Zalar, Uniform Primeness of Classical Banach Lie Algebras of Compact Operators, Publ. Math. Debrecen, to appear.

Michael Elliott,

Department of Pure Mathematics,

Queen's University Belfast,

Belfast BT7 1NN, No. Ireland

m.elliott@qub.ac.uk

Received on 29 November 2001. 